
internationales

vol. $32-n^{\circ} 1 \mid 2016$

Les migrations des Roms roumains en Europe

\title{
Les migrations des Roms roumains en Europe : politiques d'inclusion, stratégies de distinction et (dé)construction de frontières identitaires
}

The Migrations of Romanian Roma in Europe: Politics of Inclusion, Strategies of Distinction and (De)construction of Identity Boundaries

Las migraciones de gitanos rumanos en Europa: políticas de inclusión, estrategias de distinción y (de)construcción de los límites de identidad

Mihaela Nedelcu et Ruxandra-Oana Ciobanu

\section{OpenEdition}

\section{Journals}

Édition électronique

URL : https://journals.openedition.org/remi/7584

DOI : 10.4000/remi.7584

ISSN : $1777-5418$

Éditeur

Université de Poitiers

Édition imprimée

Date de publication : 1 mars 2016

Pagination : 7-17

ISBN : 979-10-90426-27-6

ISSN : 0765-0752

Référence électronique

Mihaela Nedelcu et Ruxandra-Oana Ciobanu, «Les migrations des Roms roumains en Europe politiques d'inclusion, stratégies de distinction et (dé)construction de frontières identitaires », Revue européenne des migrations internationales [En ligne], vol. 32 - $n^{\circ} 1$ | 2016, mis en ligne le 01 mars 2016, consulté le 14 avril 2022. URL : http://journals.openedition.org/remi/7584 ; DOI : https://doi.org/ 10.4000/remi.7584 


\section{Éditorial}

\section{Les migrations des Roms roumains en Europe : politiques d'inclusion, stratégies de distinction et (dé)construction de frontières identitaires}

\section{Mihaela Nedelcu ${ }^{1}$ et Ruxandra Oana Ciobanu ${ }^{2}$}

Depuis la chute du communisme en décembre 1989, la migration en provenance de Roumanie fait régulièrement la "Une " des médias internationaux quand il s'agit d'incriminer une présence incommodante et indésirable de migrants roms dans les grandes villes d'Europe. Qu'il s'agisse d'actes d'incivilité ou de (petite) criminalité, d'activités économiques illicites ou dévalorisantes, de modes de vie marqués par une pauvreté extrême ou de coutumes jugées incompatibles avec la culture occidentale, la visibilité de ces migrants agace. Elle donne lieu à des réactions de fort rejet, dans I'opinion publique et les discours politiques. Elle se traduit parfois dans des mesures administratives extrêmes, comme ce fut le cas, par exemple, des expulsions collectives de France de Roms roumains et bulgares en 2010. Elle alimente également un imaginaire de l'altérité qui nourrit la peur ancestrale de l'Autre, donnant du grain à moudre aux mouvements ultranationalistes en Europe, générant une forme de " persécution transnationale " (Bergeon, 2010); cela notamment quand l'Autre (non-national) ne se distingue plus du Nous (européen). Car, en effet, une large majorité de migrants roms sont des citoyens européens originaires des pays de l'Europe centrale et orientale et, plus particulièrement, de Roumanie.

En réalité, les migrants en provenance de Roumanie ont des profils très divers et ont déployé des modèles migratoires complexes (Diminescu, 2003 ; Potot, 2007 ; Michalon, 2003 ; Nedelcu, 2009 ; Michalon et Nedelcu, 2010 ; Ciobanu 2010 et 2015 ; etc.). Notamment, les flux migratoires se sont développés en plusieurs étapes, au gré des changements dans les politiques migratoires, du développement de réseaux et d'opportunités économiques à l'origine et à destination (Michalon et Nedelcu, 2010). En ce qui concerne les Roms roumains,

1 Professeure associée, Institut de Sociologie, Université de Neuchâtel, Faubourg de I'Hôpital 27, 2000 Neuchâtel, Suisse ; mihaela.nedelcu@unine.ch

2 Chercheuse postdoctorante Marie Curie, Centre interfacultaire de gérontologie et d'études des vulnérabilités, Université de Genève, 54 route des Acacias, 1227 Carouge (GE), Suisse ; oana.ciobanu@ unige.ch 
leurs motivations et stratégies migratoires présentent de fortes similarités avec celles des Roumains (Vlase et Preoteasa, 2012 ; Reyniers, dans ce numéro) et les parcours et modèles migratoires des uns et des autres se rejoignent souvent. Pour autant, la migration des Roms roumains ne peut pas être réduite à une simple composante de la migration roumaine.

Précisément, la catégorie "Rom migrant ", une " chimère ethno-juridique " comme le rappelle Bergeon dans ce numéro en citant Coquio et Poueyto (2014), validée et utilisée dans les discours politiques européens et nationaux, singularise une " migration rom " transversale aux catégories nationales. La " question rom " (Olivera, 2011), au-delà d'être une cible médiatique, s'est constituée en point central des agendas nationaux et internationaux relatifs aux questions migratoires, mais aussi aux politiques européennes d'inclusion sociale ${ }^{3}$. La minorité ethnique rom, transnationale par essence, est " devenue la "patate chaude" de la politique européenne " (Kropp et Striethorst, 2012). Par ailleurs, I'introduction de la libre circulation au sein de I'Union européenne (UE) a mis en exergue les failles d'une politique migratoire à deux vitesses; le droit à la mobilité de cette population étiquetée a priori de "nomade " ${ }^{4}$ étant contesté de facto à travers différentes formes de discrimination. Cette politique crée la controverse à l'intérieur de I'UE et des sociétés civiles des pays membres. En même temps, elle pointe sur la nécessité de non seulement repenser les frontières étatiques intra-européennes mais aussi d'interroger les défis que posent les frontières ethniques et culturelles au sentiment (anti)européen.

Travaillant nous-mêmes sur les questions migratoires roumaines depuis une quinzaine d'années, l'amalgame persistant entre Roms et Roumains dans les discours publics à l'égard des migrations en provenance de Roumanie nous a souvent interpellées et a généré l'idée de ce numéro spécial. Par ailleurs, les clivages catégoriels et les confusions nominatives sont complexes; les difficultés de définir les migrants roms roumains en référence aux Tsiganes autochtones et aux autres groupes roms revenant de manière récurrente dans la littérature (Csepeli et Simon, 2004 ;Tremlett, 2009 ; Bunescu, 2014 ; Cingolani, dans ce numéro). II nous semble alors nécessaire d'analyser la co-construction, par le haut et par le bas, exogène et endogène, d'une catégorie qui s'actualise davantage dans les pratiques et les interactions sociales que dans une rhétorique identitaire, juridique ou médiatique. Comme les contributions à ce numéro spécial le montrent, les "Roms migrants " de Roumanie constituent un groupe hétérogène, dont la diversité est à comprendre à partir d'une constellation de réalités migratoires et à travers de multiples stratégies de distinction qui

\footnotetext{
3 L’Union européenne a développé plusieurs stratégies globales visant l'inclusion de cette minorité (par exemple, La Décennie de I'inclusion des Roms 2005-2015, La Plateforme européenne pour l'inclusion des Roms ou encore Le Cadre européen pour les stratégies nationales d'intégration des Roms jusqu'en 2020). Les politiques nationales d'inclusion s'inspirent ou sont dictées le plus souvent par ces stratégies.

4 Cette représentation est toutefois stéréotypée et discutable ; selon le rapport « La situation des Roms en Europe : circulation et migrations " de la Commission des migrations, des réfugiés et des personnes déplacées du Conseil de l'Europe (http://www. romeurope.org/IMG/pdf/Rapport_Conseil_de_I_Europe_juin_2012.pdf, consulté le 2 décembre 2015), moins de $20 \%$ des Roms sont nomades, c'est-à-dire mènent encore une vie itinérante ou semi-itinérante. Par ailleurs, bien qu'ils sont souvent perçus comme des étrangers ou venant de l'étranger, une écrasante majorité d'entre eux possèdent la nationalité du pays où ils vivent.
} 
leur permettent de se différencier des Roumains "gadjos ", d'autres groupes " tsiganes " dans les pays d'accueil et d'autres " neam " (ethnies roms).

\section{Comprendre le problème u à la source " : la minorité rom en Roumanie}

Le recensement roumain de 2011 montre que la population résidente permanente en Roumanie est ethniquement plutôt homogène, avec 88,9 \% de Roumains, suivi par 6,5\% d'ethniques hongrois et 3,3\% de Roms. Ces derniers sont représentés par 621576 individus, dont plus de la moitié a déclaré le roumain comme langue maternelle, contre environ $40 \%$ la langue romani et $5,3 \%$ la langue hongroise ${ }^{5}$. Environ deux tiers d'entre eux vivent en milieu rural, tandis qu'un tiers se retrouvent dans des villes, le plus souvent regroupés en périphéries urbaines, dans des habitats précaires (Giurca, 2012 ; Tarnovschi, 2012). Ces chiffres sont à traiter avec prudence si on prend en compte l'analyse faite par Bunescu (2014) qui souligne que le nombre de Roms déclarés dans les recensements basés sur l'auto-identification est largement inférieur à celui issu d'estimations utilisant l'hétéro-identification. Selon cette auteure, "Roma in Romania often prefer to identify themselves as Romanians or Hungarians for fear of discrimination. Therefore, it is considered that some percentages that account for Romanian and Hungarian populations in Romania are in fact Roma " (Bunescu, 2014 : 49). Ils seraient ainsi 1,5 million, soit 6,7 \% de la population totale en Roumanie. Cela suggère que les Roms roumains mettent en place des stratégies de distinction et d'appartenance leur permettant d'échapper à la discrimination sur des bases ethniques. Néanmoins, on peut aussi penser, comme Lièvre le montre dans sa contribution à ce numéro spécial, qu'une fraction de cette minorité s'identifie en tant que Roms roumanisés, en partie suite à un processus de sédentarisation, voire d'assimilation, initié dans la période communiste (Tesăr, 2011).

Les conditions de vie et la situation économique des Roms sont très précaires ; cette minorité a été touchée dans une plus grande proportion que les autres groupes ethniques par la transition post-communiste vers une économie de marché, le chômage de longue durée et l'occupation des segments les plus fragiles du marché du travail (Reynier, 2001 ; Zamfir et Preda, 2002 ;Tarnovschi, 2012). Par ailleurs, il s'agit d'un groupe social, particulièrement vulnérable, qui cumule des " handicaps " favorisant l'exclusion (Fleck et Rughinis, 2008 ; Tarnovschi, 2012 ; Giurca, 2012). Le niveau d'éducation des Roms est peu élevé, la participation à l'école étant de 15 à $40 \%$ inférieure à la moyenne nationale selon les niveaux de formation (cycle primaire, gymnase, lycée) et un nombre important d'entre eux vivent des situations inquiétantes de non-scolarisation ou d'abandon scolaire, principalement en raison d'une précarité économique extrême (Zamfir et Preda, 2002). Une étude comparative des situations socioéconomiques des Roms en Roumanie, Bulgarie, Italie et Espagne montre également

5 Recensement roumain 2011, [en ligne]. URL : http://www.recensamantromania.ro/ rezultate-2/;Tableau 11 : Population résidente permanente selon ethnie et langue maternelle, par catégorie de localité ( "Populatia stabila dupa etnie si limba materna - catégorii de localitati »). Les questions du recensement relatives à l'appartenance ethnique, la langue maternelle et la religion font recours à I'auto-identification. 
que les Roms roumains connaissent le taux le plus important d'analphabétisme (25\%) (Pamparov et Kabakchieva, 2012). S'ajoute à cela un état de santé ainsi que des conditions d'habitat, d'accès à l'eau et à l'électricité préoccupants en termes de santé publique ; l'ensemble renforçant le " cercle vicieux de la pauvreté " (Giurca, 2012).

Face à cette situation, sous l'impulsion de I'UE, la minorité rom est devenue une cible de choix dans les politiques nationales d'inclusion des minorités après 1990. L'État roumain a développé plusieurs programmes spécifiques, culminant avec la "Stratégie du gouvernement roumain d'inclusion des citoyens roumains appartenant à la minorité rom pour la période 2011-2020 ", avec des actions sectorielles dans les domaines de l'éducation, de l'emploi, de la santé, du logement, de la culture et de l'infrastructure sociale; actions dans lesquelles les ONG jouent un rôle prépondérant (Giurca, 2012). Cependant, l'implémentation de ces politiques publiques se heurte à de nombreuses difficultés, notamment liées à un manque de coordination institutionnelle entre les niveaux local, régional et national, à une corrélation déficitaire entre l'offre des services sociaux et les besoins réels de cette population ainsi qu'à une connaissance insuffisante des mécanismes socioculturels à l'origine des processus de discrimination et de victimisation des Roms en Roumanie (Giurca, 2012).

En même temps, à l'intérieur de cette minorité on distingue des trajectoires sociales d'une grande complexité et diversité ; les Roms roumains ne constituent pas une entité sociale monolithique, et leurs réactions face à l'exclusion se construisent en fonction de ressources culturelles et matérielles propres. Par ailleurs, la diversité des stratégies (dont la migration internationale fait partie) qu'ils développent pour améliorer leur vie et échapper à la discrimination prouve qu'il faudrait déconstruire leur enfermement dans l'image "victimisante " d'une minorité assistée, et s'intéresser davantage à leur capacité d'agency (Vlase et Voicu, 2014) comme levier de l'intégration.

\section{La migration des Roms (roumains) en Europe}

L'adhésion de la Roumanie et de la Bulgarie à I'UE en 2007 a entraîné une grande inquiétude politique quant à l'ampleur que pourrait prendre la migration des Roms ressortissants de ces pays vers les autres pays européens. Cette inquiétude fut redoublée avec la détérioration générale de l'image des migrants roms, en particulier après 2008, quand ce groupe a été projeté comme un problème de sécurité en France (voir Lurbe i Puerto, dans ce numéro), et que plusieurs expulsions ont eu lieu en France et en Italie, en dépit de la législation européenne (Kropp et Striethorst, 2012).

Dans ce contexte, on constate un foisonnement d'études et de publications récentes concernant les migrations des Roms. Trois numéros spéciaux leur ont été dédiés depuis 2012. Le premier, sous la direction de Sigona et Vermeersch (2012), se focalise sur les deux types de politiques - migratoires et de protection des minorités ethniques - qui influencent les mobilités des Roms et leur image dans les discours publics. Le deuxième, dirigé par Tremlett et al. (2014), discute la question de la reconnaissance ethnique et de l'identification externes au groupe, engageant une réflexion sur la terminologie et les méthodes utilisées 
dans l'étude des migrants roms. Le dernier, coordonné par Doytcheva (2015), traite la question de la construction sociale de la figure collective des Roms, processus dans lequel se conjuguent modèles nationaux de diversité, représentations institutionnelles, idéologies néolibérales et discours politiques; il est présenté en détail dans ce volume par son éditrice, dans une note de synthèse fort intéressante. Ces trois numéros spéciaux rassemblent des travaux sur différents groupes nationaux de Roms dans plusieurs pays de destination et mettent en évidence l'articulation de plusieurs échelles d'analyse (locale, nationale, supranationale).

Par ailleurs, la migration des Roms roumains plus spécifiquement a fait l'objet de plusieurs études et publications récentes (Tesăr, 2011 ; Nacu, 2012 ; Vlase et Preoteasa, 2012 ; Pantea, 2013 ; Vlase et Voicu, 2014 ; etc.). Certaines d'entre elles adoptent une approche comparative et permettent de saisir l'impact des contextes dans les pays d'origine et les pays de destinations sur les trajectoires de ces migrants. De ce fait, Pamporov et Kabakchieva (2012), ainsi que Vlase et Preoteasa (2012), se basant sur une recherche menée sur les Roms en Roumanie, Bulgarie, Italie et Espagne, constatent des similitudes avérées dans les processus de discrimination et d'inclusion des minorités roms dans ces pays.

Sur un registre plus spécifique, d'autres études soulignent l'augmentation de la participation des femmes à ces flux migratoires (Vlase et Preoteasa, 2012 ; Pantea, 2012 ; Bergeon, dans ce numéro), même si l'émigration des femmes reste fortement dépendante des régimes de genre dans la communauté d'origine, se traduisant entre autres par un travail de maintien de la respectabilité et d'évitement d'un stigma (Pantea, 2012). Elles mettent également en évidence l'importance des réseaux familiaux et communautaires dans la mise en œuvre du projet migratoire (Tesăr, 2011 ; Vlase et Preoteasa, 2012 ; Pantea, 2013) ; un projet qui reste une réponse à l'absence d'opportunités économiques (Reyniers, 2001) et aux besoins d'améliorer la qualité de vie et de trouver des places de travail (Vlase et Preoteasa, 2012). Dans une analyse plus nuancée des contraintes et des opportunités de migration des Roms roumains, basée sur des données quantitatives du Baromètre de I'Inclusion Rom et des entretiens approfondis, Vlase et Voicu (2014) montrent que le genre, la religion et l'appartenance de neam représentent certes des facteurs qui influencent l'impact des contraintes structurelles sur la décision de migration des Roms, mais ces auteurs mettent également en évidence leur agency, notant finalement que les Roms ne sont pas " a passive population, but active agents able to act and struggle to improve their living conditions and their relationships with the public institutions " (2014: 2433).

Le présent numéro s'inscrit dans ce débat très actuel et très fourni sur les " migrations rom " en proposant une lecture renouvelée des stratégies de migration et d'inclusion des Roms roumains, basée sur le rôle des processus $d^{\prime}$ identification et d'auto-identification en tant que facteurs qui peuvent agir à la fois comme un frein ou un levier à la mobilité et/ou l'intégration de ces populations. Comme le souligne Bunescu, si l'identité rom est en permanence négociée par les Roms en rapport avec les non-Roms, on peut distinguer plusieurs types d'identifications " that can be located on a continuum from extrovert, performed, instrumental, and ascribed to introvert, denied, felt, and self-ascribed " (Bunescu, 2014 : 194). 
À travers ce prisme et en croisant perspectives institutionnelles et approches ethnographiques, les contributions à ce numéro participent d'une déconstruction de la catégorie " migrant rom roumain " dont I'utilisation n'est pas sans implications identitaires, relationnelles et politiques. Ainsi, visant à comprendre les "frontières " qui délimitent la migration des populations rom au sein de la migration roumaine, mais qui agissent aussi à l'intérieur de la minorité rom elle-même, ce numéro spécial tente de répondre aux questions suivantes : comment sont construites ces frontières par le haut (politiques formelles) et par le bas (stratégies de différenciation, réseaux sociaux et identités culturelles) ? Comment se positionnent et $\mathrm{s}^{\prime}$ (auto)identifient les migrants roms roumains dans les sociétés d'origine et d'accueil ? Comment s'entrecroisent ethnicité, identité, genre et citoyenneté dans leurs stratégies migratoires et identitaires?

En ouverture de ce numéro et quinze ans après avoir fait état de l' " exemple roumain " (Reyniers, 2001), la contribution d'Alain Reyniers, intitulée "Mouvements migratoires et circulation des Roms roumains en Europe ", propose un bilan des migrations internationales des Roms originaires de Roumanie depuis la chute du communisme en 1989. Après une brève discussion de la multiplicité des significations linguistique, ethnique et sociale du vocable "Rom " qui reste fortement politisé, cet auteur situe les mouvements récents des migrants roms dans une logique migratoire qui remonte au Xle siècle. II souligne aussi que, depuis la fin de la Seconde Guerre mondiale, les flux migratoires des Roms se sont développés de manière non univoque, à travers de multiples mobilités intra-régionales touchant la plupart des pays des Balkans. Cependant, leur visibilité dans les pays de l'Ouest s'accroit à partir de 1990. Poussés par le manque de perspectives sur le marché du travail et victimes d'exclusion sociale en Roumanie, les Roms font de I'asile politique une porte d'entrée privilégiée en Europe pendant la décennie 1990. Leurs mobilités se sont déployées dans le sillage de la dynamique européenne des politiques migratoires à l'égard des ressortissants roumains en général, avec l'émergence de I'espace Schengen et I'adhésion de la Roumanie à I'UE comme tournants significatifs de ces politiques. En même temps, la population rom est fortement hétérogène, se caractérisant par une méfiance entre les groupes nationaux et en dehors des réseaux familiaux ou communautaires fermés; ce qui mène à une double perception des Roms : de l'extérieur comme un groupe compact, marqué toutefois à l'intérieur par de fortes " singularités ethniques ".

En ce sens, Marion Lièvre procède à une déconstruction du terme "Rom " en analysant l'usage que font les Roms roumains présents à Montpellier des catégories endo-ethnonymiques et hétéro-ethnonymiques. L'ethnographie des frontières ethniques et culturelles qu'elle propose permet de mettre en évidence les processus de différenciation entre les groupes de Roms roumains, dans les lieux de destination. Ces processus d'auto-désignation et de désignation des autres reflètent des stratégies de distinction sociale intra et extra-groupe qui constituent des ressources importantes en contexte migratoire. En effet, analysant comment on devient Roms roumanisés, Ciurari, Ursari ou Bogdanesteni, I'auteure montre que la façon dont les Roms mobilisent eux-mêmes ces catégories traduit des logiques d'identification et de différenciation qui contribuent à la "fabrique " des frontières entre ces groupes. Dans les luttes symboliques entre ces derniers s'entremêlent rapports sociaux, appartenances ethniques et caractéristiques culturelles qui témoignent d'un rapport différencié aux tradi- 
tions " roms " qui occupent une place significative dans l'affirmation de " nous ethniques " distincts. L'auteure conclut en soulignant que ces rapports de domination symbolique entre les groupes roms montpelliérains reflètent néanmoins la réification d'une " authenticité tsigane ", quelque peu redéfinie à travers une vision moderne du rapport à la culture.

Les articles proposés à la suite par Pietro Cingolani et par Katìa Lurbe i Puerto étudient l'impact des programmes de relogement sur les trajectoires d'intégration des participants. Ces deux articles prolongent les réflexions sur les processus d'identification des migrants roms autour de l'articulation entre une construction identitaire par le bas, basée sur l'auto-identification, et par le haut, à travers les classifications et les désignations imposées par les administrations nationales pour identifier les groupes cibles des politiques de relogement.

À partir d'une étude anthropologique menée àTurin, en Italie, dans plusieurs sites dans le sud-ouest de la Roumanie et en se focalisant sur six études de cas spécifiques, Pietro Cingolani analyse l'intersection entre représentations institutionnelles et auto-identification, tout en tenant compte des parcours de vie et de migration, du contexte à l'origine et à destination des Roms. II montre que les relations et la cohabitation entre Roms et non-Roms dans les lieux d'origine influencent fortement la manière de s'auto-identifier des migrants roms roumains; en même temps, les migrants peuvent aussi jouer de manière instrumentale la carte de l'identité rom, afin de répondre aux définitions et attentes des institutions et ainsi tirer bénéfice des politiques sociales en Italie. De cette manière, I'auteur montre que la question identitaire est une question multi-échelle, à étudier " at different analytical levels: the local, the state, and the transnational ".

En soulignant une superposition problématique entre les catégories "Rom " et " Gens du voyage " ainsi que le rôle de la " séquence Rom de 2010 " dans la " fabrication politique " du " problème Rom " en France, Katìa Lurbe i Puerto propose une analyse des politiques urbaines et d'inclusion sociale s'adressant aux migrants roms en France à partir d'une étude de cas focalisant sur l'impact du projet Sénart. Ce projet de discrimination positive, mené de 2000 à 2007, a eu un impact considérable sur les trajectoires d'intégration des participants. Adoptant une approche d'intersectionalité contextuelle et relationnelle, l'auteure identifie trois trajectoires distinctes d'intégration. La première est celle des familles marquées par une forte socialisation dans les bidonvilles, avec un fort taux d'illettrisme et des relations intrafamiliales de pouvoir qui désavantagent les belles-filles; autant de caractéristiques qui empêchent les participants de profiter des programmes d'intégration offerts par le projet. La deuxième concerne des familles avec des parcours professionnels ininterrompus, commencés déjà en Roumanie et avec une histoire de migration multiple en Europe. Dans ces familles, les femmes travaillent et ont pu aussi bénéficier de cours de français proposés dans le cadre du projet. Dans ce cas, l'éducation des enfants occupe une place importante, représentant un instrument de mobilité sociale. La troisième réfère à la trajectoire de jeunes couples avec des enfants en bas âge, ayant accédé à des logements sociaux et dont les femmes ont pu suivre une formation secondaire en France, tandis que les hommes sont restés en situation d'illettrisme. Cependant, conservant un modèle patriarcal des tâches au sein de la famille, ces participants au projet Sénart se déclarent déçus par le projet, 
car cela ne les aurait pas aidés à s'intégrer sur le marché du travail. De manière générale, cette étude de cas analyse ainsi la construction d'une " ethnicinstrumented class " au carrefour des politiques urbaines et d'une approche sécuritaire de la migration rom en France. Elle montre aussi que le succès des programmes d'insertion sociale dépend fortement des ressources humaines, sociales et culturelles dont disposent les migrants.

En rappelant que la question de la catégorisation est sensible et qu'elle s'opère principalement dans les sphères médiatique et politique, Céline Bergeon aborde une dimension inédite de la visibilité des migrants roms de Roumanie dans l'espace public. S'attelant à déconstruire une image stéréotypée de ces migrants, elle analyse les mobilisations associatives exogènes et endogènes, c'est-à-dire " pour " et " par " les Roms, par le prisme de I'habitat et des temporalités migratoires. Tout en soulignant le rôle de la famille et des réseaux transnationaux de parenté, ainsi que la féminisation importante des flux de migration, l'auteure met en évidence l'importance de la dimension culturelle et de la mise en scène festive de leurs traditions par les migrants roms comme des modes de contestation politique et de visibilité sociale " positive ". Aussi, relevant la grande diversité dans le paysage associatif rom en Île-de-France, elle montre que les manifestations les plus visibles (souvent internationales et politisées, comme "Roma Pride ") n'ont de loin pas le même impact sur le quotidien des migrants roms que les événements festifs locaux; ce sont les manifestations locales, favorisant les contacts avec le voisinage et les rencontres interculturelles qui concourent à un changement des représentations sociales et à un apaisement des rapports sociaux.

L'analyse des modalités d'insertion des Roms dans un espace urbain ségrégué - un marché de périphérie à Nice - donne l'occasion à Ryzlène Dahhan de montrer comment, dans les interactions quotidiennes des Roms avec les autres acteurs présents dans cet espace, se mettent en scène des processus de différenciation et de hiérarchisation ethniques. Basé sur une enquête ethnographique combinant observations participantes et non participantes avec des entretiens de type conversation, cet article dresse d'abord une description détaillée de l'inscription des activités commerciales légales et illégales dans l'espace, associée à la coprésence de commerçants de différentes origines (notamment Roms et Nord-Africains). II met ensuite en évidence comment cette inscription définit des frontières spatiales et relationnelles entre les groupes qui se côtoient et se partagent le marché illégal, le marché forain et la galerie marchande à I'Oued; reflétant des logiques d'inclusion et d'exclusion dans lesquelles se négocient légitimité sociale et rapports de pouvoir. Ainsi, la dimension fonctionnelle et la spécificité des activités commerçantes des vendeurs roms, ainsi que la présentation de soi de ces derniers dans ce contexte, assurent leur légitimité sur le marché illégal et relèguent la marque ethnique au second plan. Tandis qu'en dehors de ce périmètre, leur présence gêne et une forte différenciation ethnique opère à travers des interactions marquées par la méprise et l'assignation à une catégorie minorisée, reflétée notamment dans l'interpellation de cette population par I'appellation Roms/Romani.

Dans une perspective transnationale, la contribution de Norah BenarroshOrsoni donne un éclairage inédit du fonctionnement des réseaux de parenté et des rapports familiaux à la fois à proximité et à distance, en mettant en exergue 
le rôle que joue la communication téléphonique à ce propos. À Montreuil, en France, les ménages des Roms migrants bénéficiant d'un logement social, et donc d'un habitat permettant une ligne téléphonique fixe, deviennent les agents clés d'une sociabilité transnationale ; autour d'eux gravitent d'autres migrants moins chanceux qui utilisent les facilités communicationnelles dont ils bénéficient en France. Du côté roumain, les proches parents se sont eux aussi rapidement outillés en téléphones fixes et mobiles. Profitant d'offres commerciales très avantageuses et de communications quasi-gratuites sur les lignes fixes, les communications entre ici et là-bas se sont multipliées, bien qu'elles prennent des voies plutôt spécifiques : les conversations se font plutôt en groupe, souvent entre femmes, et révèlent des fonctions bien précises. Elles servent à renforcer le contrôle social familial et à rappeler au migrant ses "devoirs " envers les parents restés au pays; ces derniers réitérant constamment des demandes concernant l'envoi d'argent et de biens spécifiques. En même temps, tout en contribuant à maintenir des relations plus ou moins conflictuelles, ces communications collectives participent de la cohésion sociale communautaire. Elles permettent aussi de renforcer le sentiment d'une double appartenance.

À cette collection d'articles s'ajoute la note de synthèse signée par Milena Doytcheva. Le dossier paru en 2015 sous sa direction dans Confluences Méditerranée aborde la question rom à travers I'analyse des " cadrages individuels et plus politiques des démarches d'identification et d'appartenance collective ". Avec l'avantage de s'appuyer sur des résultats d'enquêtes portant sur les Gitanos en Espagne, les Romanlar en Turquie, les Roms de Bulgarie, etc., ce dossier représente une contribution significative à la compréhension des processus de catégorisation et leur importance dans la construction sociale des figures collectives des Roms et Tsiganes en Europe méditerranéenne. Dans la note de synthèse qu'elle nous propose, Milena Doytcheva souligne la dimension revendicative de l'appellation "Rom ", endonyme supposé unificateur et mobilisateur. En même temps, si cette catégorie participe d'une stratégie endogène de " destigmatisation ", ses usages dans les pratiques, les représentations et les politiques en faveur des Roms peuvent générer différentes situations de mise à l'écart, de racialisation et de coercition ; les modèles d'intégration nationale et les situations locales constituant d'importants facteurs discriminants. Ainsi I'analyse comparative et historique des cas français, espagnol et italien montre que l'étude des migrations récentes des Roms doit s'intéresser systématiquement au "système d'inégalités "encastrées" et structurantes " qui affecte ces migrants. Mais elle rappelle aussi l'importance de pratiquer un " "décentrement" du regard porté sur ces objets " et d'étudier ces groupes spécifiques, d'une part, dans le contexte plus large des migrations internationales et des relations interethniques, ainsi que des interdépendances globales qui les constituent ; et, d'autre part, en se focalisant davantage sur les relations au quotidien entre ces groupes migrants et les populations locales.

Le présent recueil d'articles sur les migrations des Roms roumains renforce cette idée, en soulignant qu'il s'agit d'une catégorie qui s'actualise sans cesse dans les pratiques et les interactions sociales. II documente la complexité des processus identitaires et de catégorisation dont la compréhension nécessite d'articuler constamment logiques et stratégies d'auto-identification et d'hétéroidentification, à différentes échelles (locale, nationale, transnationale et européenne). En même temps, il s'agit à chaque fois de ne pas oublier l'incidence des 
contextes socioculturels et politiques dans lesquels ces processus se déroulent à ces différentes échelles - sur la construction de (nouvelles) figures collectives : à I'heure où ce numéro paraîtra, la " question rom " se sera fait voler la vedette, remplacée par la préoccupante crise des réfugiés en Europe et la menace terroriste mondiale - l'une comme l'autre nous rappelant, une fois de plus, que nous vivons dans un monde de mobilités et d'interdépendances inextricables.

\section{Références bibliographiques}

Bergeon Céline (2010) Les Roms roumains en France. Entre politiques migratoires et politiques de non-accueil, Revue d'Études Comparatives Est-Ouest, 41 (4), pp. 197-211.

Bunescu loana (2014) Roma in Europe: The Politics of Collective Identity Formation, Farnham, Ashgate, 228 p.

Ciobanu Ruxandra Oana (2015) Multiple Migration Flows of Romanians, Mobilities, 10 (3), pp. 466-485.

Ciobanu Ruxandra Oana (2010) The Thin Line Between Family Migration and Network Migration. Family Stories of the Romanian Migration to Spain, Revue d'Etudes Comparatives Est-Ouest, 41 (4), pp. 125-148.

Coquio Catherine et Poueyto Jean-Luc (2014) Roms, Tsiganes, Nomades. Un malentendu européen, Paris, Khartala, 676 p.

Csepeli György and Simon Dávid (2004) Construction of Roma identity in Eastern and Central Europe: Perception and self-identification, Journal of Ethnic and Migration Studies, 30 (1), pp. 129-150.

Diminescu Dana (Éd.) (2003), Visibles mais peu nombreux. Les circulations migratoires roumaines, Paris, Éditions de la MSH, 340 p.

Doytcheva Milena (2015) Roms et Tsiganes en Europe méditerranéenne : I'actualité d'une question, Confluences Méditerranée, 2 (93), pp. 9-25.

Fleck Gábor and Rughiniş Cosima (Eds.) (2008) Come Closer: Inclusion and Exclusion of Roma in Present-Day Romanian Society, Bucarest, Human Dynamics, $266 \mathrm{p}$.

Giurca Diana (Ed.) (2012) Incluziunea romilor din Romania: politici, institutii, experiente. Roma inclusion in Romania: Policies, institutions and examples, Constanta, Editura Dobrogea, 140 p.

Kropp Manuela et Striethorst Anna (2012) La migration des Roms au sein de I'Union européenne. Une minorité ethnique est devenue la "patate chaude " de la politique européenne, Transform !, 10, pp. 191-204.

Michalon Bénédicte (2003) Migrations des Saxons de Roumanie en Allemagne. Mythe, interdépendance et altérite dans le " retour ", Thèse en géographie, Université de Poitiers, 582 p.

Michalon Bénédicte et Nedelcu Mihaela (2010) Introduction : Histoire, constantes et transformations récentes des dynamiques migratoires en Roumanie, Revue d'Etudes comparatives Est-Ouest, 41 (4), pp. 5-27. 
Nacu Alexandra (2012) From Silent Marginality to Spotlight Scapegoating? A Brief Case Study of France's Policy Towards the Roma, Journal of Ethnic and Migration Studies, 38 (8), pp. 1323-1328.

Nedelcu Mihaela (2009) Le migrant online. Nouveaux modèles migratoires à l'ère du numérique, Paris, L'Harmattan, 326 p.

Olivera Martin (2011) La fabrique expert de la "question rom " : multiculturalisme et néolibéralisme imbriqués, Lignes, 34, pp. 104-118.

Pamporov Alexey and Kabakchieva Petia (2012) Social inclusion and discrimination of Roma in four EU countries, in Daniela Tarnovschi Ed., Roma from Romania, Bulgaria, Italy and Spain between Social Inclusion and Migration: Comparative study, Constanta, Editura Dobrogea, pp. 17-32.

Pantea Maria-Carmen (2013) Social Ties at Work: Roma Migrants and the Community Dynamics, Ethnic and Racial Studies, 36 (11), pp. 1726-1744.

Pantea Maria-Carmen (2012) From "Making a Living" to "Getting Ahead": Roma Women's Experiences of Migration, Journal of Ethnic and Migration Studies, 38 (8), pp. 1251-1268.

Potot Swanie (2007) Vivre à l'Est, travailler à l'Ouest. Les routes roumaines de I'Europe, Paris, L'Harmattan, $232 \mathrm{p}$.

Reyniers Alain (2001) Migrations et déplacements des Roms de I'Est : I'exemple roumain, Confluences Méditerranée, 38 (3), pp. 59-66.

Sigona Nando and Vermeersch Peter (2012) Editors' Introduction. The Roma in the New EU: Policies, Frames and Everyday Experiences, Journal of Ethnic and Migration Studies, 38 (8), pp. 1189-1193.

Tarnovschi Daniela (Ed.) (2012) Roma from Romania, Bulgaria, Italy and Spain between Social Inclusion and Migration: Comparative study, Constanta, Editura Dobrogea, $88 \mathrm{p}$.

Tesăr Cătălina (2011) "Tigan bun traditional" în România, cerșetor de-etnicizat în străinătate. Politici ale reprezentării publice și etica muncii la romii cortorari, in Stefánia Toma şi László Fosztó Eds., Spectrum. Cercetări sociale despre romi, Cluj Napoca, ISPMN, pp. 281-312.

Tremlett Annabel (2009) Bringing Hybridity to Heterogeneity: Roma and the question of difference in Romani studies, Romani Studies, 19 (2), pp. 147-168.

Tremlett Annabel, McGarry Aidan and Agarin Timofey (2014)The Work of Sisyphus: Squaring the Circle of Roma Recognition, Ethnicities, 14 (6), pp. 727-736.

Vlase Ionela and Preoteasa Ana Maria (2012) Roma Migrants from Bulgaria and Romania. Migration Patterns and Integration in Italy and Spain 2011, in Daniela Tarnovschi Ed., Roma from Romania, Bulgaria, Italy and Spain between Social Inclusion and Migration: comparative study, Constanta, Editura Dobrogea, pp. 65-87.

Vlase Ionela and Voicu Mălina (2014) Romanian Roma migration: The interplay between structures and agency, Ethnic and Racial Studies, 37 (13), pp. 2418-2437.

Zamfir Cătălin şi Preda Marian (Eds.) (2002) Romii în România, Bucureşti, Editura Expert, $355 \mathrm{p}$. 